\title{
Efficient ant colony optimization for computer aided molecular design: case study solvent selection problem
}

(1)

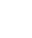

$$
\text { Center for Uncertain Systems: Tools for Optimization \& Management (CUSTOM), }
$$$$
\text { Vishwamitra Research Institute, Crystal Lake, IL } 60012
$$$$
\text { berhane@vri-custom.org, Urmila@vri-custom.org }
$$ 


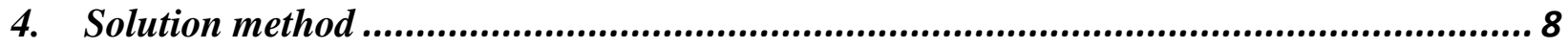

\section{Introduction}

Solvents are used for a variety of purposes in process industries. They are extensively used as process materials, as extracting agents, and as process liquids in process industries, pharmaceutical industries, and solvent based industries. Waste solvents are main source of pollution to air, water, and soil. Therefore, it is empirical to use environmentally benign solvents without compromising the process performance. Moreover, there are some solvents that must be eliminated because of environmental and health effects and regulatory requirements (Karunanithi et al., 2005, Kim \& Diwekar, 2002bc, Xu \& Diwekar, 2005). The environmental awareness, the strict legislation and the need for high performance solvents have resulted in the search for less toxic and environmentally benign solvents and solvent formulations that have improved performance characteristics. Several methodologies have been developed for solvent selection over the years. The first approach uses traditional laboratory synthesis and test methodology to find promising solvents. This method can provide reliable and accurate results, but in many cases this approach is limited by cost, safety, and time constraints. The second approach is to screen the property database. Though, the screening of the database is the most common and simple method, it is limited by size and accuracy of the database. These methods are usually expensive and time-consuming. 
Solvent selection based on computer aided molecular design (CAMD) is fast emerging systematic tool for efficient and reliable design of candidate solvents from their fundamental building blocks (Jorge Marrero, 2001, Karunanithi et al., 2005, Kim \& Diwekar, 2002bc, Xu \& Diwekar, 2005). Beyond the solvent selection, the CAMD technique is practiced with great success in different disciplines such as pharmaceutical process designs (Gernaey and Gani, 2009), polymer design (Satyanarayana et al., 2009), and bioethanol production (AlvaradoMorales et al. 2009). CAMD is generating large number of structural molecules with desired properties from a small set of structural groups (building blocks). CAMD is the reverse use of the group contribution method. Different solution strategies are implemented to solve CAMD techniques: heuristic numeration (Hostrup et al. 1999, Li et al., 2002), knowledge based technique (Harper \& Gani, 2000, Yamamoto \& Tochigi, 2008), molecular property clusters with algebraic equations (Chemmangattuvalappil et al. 2009, Eljack \& Eden, 2008, Kazantzi et al., 2007) and optimization-based methods (Karunanithi et al., 2005, Samudra and Sahinidis, 2005, Diwekar \& Shastri 2011, Ostrovsky et al., 2002). problem (MINLP) that seeks to optimize the desired properties of the solvent molecules subject

In the optimization approaches, because of the nonlinearity behavior of the UNIFAC model, the CAMD for solvent selection is formulated as a mixed integer nonlinear programming to molecular design feasibility rules. To solve the MINLP formulation of the CAMD problems, different optimization methods have been proposed: decomposition methods that use local optimizers for the NLP sub-problem (Odele and Macchietto, 1993, Harper et al., 1999, Karunanithi et al., 2005, Karunanithi et al., 2006), global optimization (Ostrovsky et al., 2002, Samudra and Sahinidis, 2005), interval analysis (Achenie \& Sinha, 2003), and dynamic optimization (Apostolos Giovanoglou et al., 2003). Recently, probabilistic methods such as simulated annealing (Kim \& Diwekar, 2002a, b, c), genetic algorithms (Cheng \& Wang, 2008, Diwekar \& Xu, 2005, Xu \& Diwekar, 2005) are adopted as an alternative to the local optimization strategies to find better solutions.

Ant colony optimization (ACO) algorithm proposed by (Dorigo, 1992) is a metaheuristic optimization strategy that can provide a viable alternative to solve the MINLP of the CAMD problem. Although in recent years, there has been a significant research interest in developing and implementing for different applications, to the authors knowledge, there is no work in the 
literature that implement efficient ant colony (AECO) algorithm to solve the MINLP CAMD problems.

The algorithm implemented in this work to solve the solvent selection problem combines the CAMD and the EACO algorithm proposed by (Gebreslassie \& Diwekar, 2015) as shown in Figure 1. The algorithm parameters, the UNIFAC building block groups and their properties such as the volume and surface area parameters, and the interaction parameters between groups are first introduced. These UNIFAC groups are uniquely designed to generate all possible molecules by exploring all possible combinations. Using the group combinations from this set of groups, solvent molecules are generated. For example, as shown in the algorithm, ethanol $\left(\mathrm{CH}_{3} \mathrm{CH}_{2} \mathrm{OH}\right)$ is generated from the $\mathrm{CH}_{3}, \mathrm{CH}_{2}$, and $\mathrm{OH}$ groups. The number of combinations can be reduced by introducing constraints from physical and chemical properties, structural feasibility as well as those from regulatory restrictions. Once molecules are generated, the properties of the molecules are predicted based on the properties of their groups using the UNIFAC model. This method can generate candidate solvents with a reasonable accuracy. The rest of the paper is organized as follows. Section 2 describes the CAMD model formulation. The optimization problem of the solvent selection under study is formulated as an MINLP model in section 3. Ant colony optimization and the proposed EACO algorithm to solve the MINLP optimization problem is presented in section 4 followed by section 5 that discusses the result. Finally, section 6 presents the concluding remarks of the paper.

\section{Solvent Selection Model Formulation}

To replace the current solvent or formulate a new one, there are several criteria and solvent properties that can be reviewed such as the solute distribution coefficient $m$, solvent selectivity $\beta$, solvent loss $S_{L}$, and physical properties like boiling point, density, viscosity, and so on.

Distribution coefficient $(m)$ : it is a measure of solvent capacity and it represents the solute distribution between the solvent and the raffinate phases. A high value of $m$ reduces the size of extraction equipment and the amount of recycling solvent. The solute distribution coefficient is estimated as by Eqn. 1 .

$$
m=\frac{\text { concentration of solute in extractive phase }}{\text { concentration of solute in raffinate phase }} \cong \frac{\gamma_{B A}^{\infty}}{\gamma_{B S}^{\infty}} \frac{M W_{A}}{M W_{S}}
$$


113 where $A, B$, and $S$ represent the raffinate (a nonpolluting molecule eg. water), solute (polluting 114 molecule eg. acetic acid), and solvent (eg. ethanol) phases, respectively. MW denotes the 115 molecular weight, and $\gamma^{\infty}$ is the infinite dilution activity coefficient.

116 Solvent selectivity $(\beta)$ : It is the ratio between the solute distribution coefficient and raffinate. It 117 estimates the ability of the solvent to selectively dissolve a solute (polluting molecule). A high 118 solvent selectivity value thus can reduce the cost of solute recovery and it is defined as shown in 119 Eqn. 2.

$$
\beta=\frac{\text { distribution coefficient of solute }}{\text { distribution coefficient of solvent }}=\frac{m_{B}}{m_{A}} \cong \frac{\gamma_{A S}^{\infty}}{\gamma_{B S}^{\infty}} \frac{M W_{B}}{M W_{A}}
$$

Solvent loss $\left(S_{L}\right)$ : it is a measure of the concentration of solvent in raffinate phase. It is the measure of the solvent loss tendency; a low solvent loss value means high selectivity toward 124 solute and determines the immiscibility between the solvent and the raffinate. It is defined as as 125 shown in Eqn. 3.

$126 S_{L}=$ concentration of solvent in raffinate phase $\cong \frac{1}{\gamma_{S A}^{\infty}} \frac{M W_{S}}{M W_{A}}$

In Eqns. 1, 2 and 3, the distribution coefficient, the solvent selectivity and the solvent loss properties are given as a function of the infinite dilution activity coefficients $\left(\gamma^{\infty}\right)$. It shows the non-ideality of the mixtures $(A-B, A-S$, and $B-S)$. If the mixture is ideal, $\gamma^{\infty}$ is close to one.

130 Otherwise, tends to be greater than one or close to zero. $\gamma^{\infty}$ is a function of groups, temperature $131 T$, pressure $P$, and concentration. There are several group contribution methods for the prediction 132 of $\gamma^{\infty}$. The most popular method is the UNIFAC group contribution method (Harper et al., 1999, 133 Hostrup et al., 1999, Kim \& Diwekar, 2002c, Xu \& Diwekar, 2005).

134 In the UNIFAC model, the activity coefficient $\left(\gamma_{i}\right)$ of a molecule $i$ have two parts: the 135 combinatorial and residual part.

$136 \quad \ln \gamma_{i}=\ln \gamma_{i}^{C}+\ln \gamma_{i}^{R}$ 
The combinatorial part reflects the volume and surface area of each molecule; hence, the volume $q_{m}$ and surface area $r_{m}$ parameters of each group $m$ in molecule $i$ are involved in estimating the combinatorial part $\left(\gamma_{i}^{C}\right)$. The residual part represents the interaction energies of the molecules; hence, the volume parameter and interaction parameters $\left(a_{m n} ; a_{n m}\right)$ between groups $m$ and $n$ in the mixture are required to predict $\gamma_{i}^{R}$. The interaction parameters can be obtained by regression of experimental data of the mixture. The infinite dilution activity coefficient of molecule $i$ in mixture is limiting activity coefficient when the concentration of molecule $i$ in the mixture tends to zero. That is, $\gamma_{i}$ is function of the volume and surface area parameters, interaction parameters, temperature, pressure, and concentration. In this paper, the 146 infinite dilution activity coefficients are calculated by the fifth revised original UNIFAC model (Hansen et al., 1991, Kim \& Diwekar, 2002c) in which the original UNIFAC model is used with new groups and a revised data table. The UNIFAC model is summarized in the following Eqns. $5,6,7$, and 8 .

$\ln \gamma_{i}^{C}=\ln \left(\frac{\Phi_{i}}{x_{i}}\right)+\frac{z}{2} q_{i} \ln \left(\frac{\theta_{i}}{\Phi_{i}}\right)+l_{i}-\frac{\Phi_{i}}{x_{i}} \sum_{j} x_{j} l_{j}$

151

$\ln \gamma_{i}^{R}=\sum_{k} v_{k}^{i}\left(\ln \Gamma_{k}-\ln \Gamma_{k}^{i}\right)$

152

$\ln \Gamma_{k}=Q_{k}\left[1-\ln \left(\sum_{m} \theta_{m} \psi_{m k}\right)-\sum_{m} \frac{\theta_{m} \psi_{k m}}{\sum_{n} \theta_{n} \psi_{n m}}\right]$

153 where

$$
\theta_{m}=\frac{Q_{m} X_{m}}{\sum_{m} Q_{m} X_{m}}, \quad \psi_{m n}=\exp \left(-\frac{a_{m n}}{T}\right), \quad \theta_{i}=\frac{q_{i} x_{i}}{\sum_{j} q_{j} x_{j}}, \quad \Phi_{i}=\frac{r_{i} x_{i}}{\sum_{j} r_{j} x_{j}}
$$

In these equations, $x_{i}$ is the mole fraction of component $i, \theta_{i}$ is the area fraction, $\Phi_{i}$ is the segment fraction, and $r_{i}$ and $q_{i}$ are measures of molecular van der Waals volume and molecular surface area, respectively. $\theta_{m}$ is the area fraction of group $\mathrm{m}, X_{m}$ the mole fraction of group $\mathrm{m}$ in the mixture, and $a_{m n}$ the group interaction parameter. The combinatorial part, $\ln \gamma_{i}^{C}$, is dependent on the sizes and shapes of the molecules, whereas the residual part, $\ln \gamma_{i}^{R}$, is dependent on group areas and group interactions. 
Normal boiling point $\left(T_{b p}\right)$ : It is estimated using the following linear prediction model (Joback, 1987).

$T_{b p}=\sum_{i}^{N_{1}} t_{a}\left(N_{2}^{i}\right)+t_{b}$

To estimate $T_{b p}$ using Eqn. 9, the boiling point group contribution parameters $t_{a}$ and $t_{b}$ are required and they are given in Table A.2 of the Appendix.

For structural feasibility of a solvent configuration, the octet rule relates the total number of free attachments of groups within the solvent molecule and the number of groups in the solvent molecule. Therefore, to determine the chemical feasibility the octet rule is used for acyclic groups as shown in Eqn. 10.

$\sum_{i}^{N_{1}} b_{i}=2\left(N_{1}-1\right)$

where $b_{i}$ is the number of free attachments in a group index $i$. Both high boiling point and low boiling point solvents are considered in the configuration.

\section{Solvent Selection Optimization Problem}

The design task is finally posed as a mixed integer nonlinear programming (MINLP) problem that seeks to maximize the solute distribution coefficient of the candidate solvent subject to structural feasibility constraint (Eqn. 10), solvent performance property constraints (Eqns. 2, 3 and 9), the UNIFAC mixture property constraints (Eqns. 4, 5, 6,7 and 8). The lower and upper limit constraints related to the number of building block groups, type and the total number of groups making up the solvent molecule are also added. The problem formulation for an acetic acid extraction (one of the case studies widely studied for the applications of CAMD Odele and Macchietto, 1993, Hostrup et. Al., 1999, Kim \& Diwekar, 2002c), which is commonly used as a process solvent or produced as a byproduct, is given in Eqn. 11. Because acetic acid can be a pollutant as well as a valuable solvent, it is desirable to minimize the discharge of acetic acid to the environment. To recycle or remove acetic acid from waste process streams, extraction process is commonly utilized. For the extraction process, one can either use high-boiling solvents or low boiling solvents (Joback, 1987, Kim \& Diwekar, 2002c) depending on the process considered. Ethyl acetate, isoamyl acetate, and isopropyl acetate are widely used in industries to 
extract acetic acid. Ethyl acetate, which is one of the common solvents for acetic acid extraction, has high $m(0.3156)$, but it unfortunately also has high $S_{L}(0.0560)$.

In this work, the high-boiling and low-boiling solvent candidates are generated by EACO algorithm and compared with candidate solvents proposed from the literature solved using other heuristic optimization strategies. The optimal high $\mathrm{m}$ and low $S_{L}$ solvent can be easily separated from the extract stream and then recycled to the extraction equipment. As shown in Eqns. 4, 5, 6, 7, and 8, the solvent selection optimization problem Eqns. 11 is formulated based on $\gamma^{\infty}$.

$$
\begin{array}{lll}
\min _{N_{1} N_{2}^{i}}- & \\
\text { s.t } & \beta \geq \beta^{\min } & \\
& S_{L} \leq S_{L}^{\max } & \\
& T_{b p}^{\min } \leq T_{b p} \leq T_{b p}^{\max } & \\
& 1 \leq N_{1} \leq 10 \\
& 1 \leq N_{2}^{i} \leq 24 \quad \forall i \in N_{1}
\end{array}
$$

The boundaries on the constraints are taken from the properties of the current practice of the solvent for acetic acid extraction (Kim \& Diwekar, 2002c). In this optimization problem, the discrete decision variables are the number of groups $N_{1}$ involved in a solvent molecule and the group index (the type of building blocks) $N_{2}^{i} ; i \in\left\{1, \ldots, N_{1}\right\}$ of that molecule. From the set of building blocks a unique solvent molecule that has high solute distribution coefficient and satisfies the selectivity, solvent loss, normal boiling point, and structural feasibility constraints can be generated.

Due to the equality (Eqns. 2-10) and inequalities such as the boundary constraints, the solvent selection problem is highly constrained optimization problem. However, the conventional ACO algorithm handles unconstrained optimization problems. In EACO algorithm, to handle constrained optimization problems Oracle penalty method (Schluter and Gerdts, 2010) is used. For details of this method please refer to (Gebreslassie and Diwekar, 2015).

\section{Solution method}

The solvent selection MINLP problem can be solved using different approaches but finding the optimal solution is not trivial. The equality constraints representing the property models are nonlinear and the gradient based methods such as Branch and Bound (BB), Generalized Bender's 
212 Decomposition (GBD), and Outer-Approximation (OA), are generally used for solving MINLP

213 problems. However, these methods have limitations whenever, the optimization problems do not 214 satisfy convexity conditions, the problems have large combinatorial explosion, or the search 215 domain is discontinuous (Diwekar \& Xu, 2005). Metaheuristic optimization strategies such as 216 simulated annealing (SA) (Kirkpatrick et al., 1983), genetic algorithm (GA) (Holland, 1975) and 217 ant colony optimization (ACO) (Dorigo, 1992) provide a viable alternative to the gradient based 218 mathematical programming techniques. Although in recent years, there has been a significant 219 research interest in developing ant colony optimization algorithms. To the best of the authors' 220 knowledge, there has not been utilized to solve the CAMD problems.

223 (Dorigo \& Stutzle, 2004). Natural ants randomly search food by exploring the area around their nest. If an ant locates a food source, while returning back to the nest, it lay down a chemical pheromone trail that marks its path. This pheromone trail will indirectly communicate with other members of the ant colony to follow the path. Over time, the pheromone will start to evaporate and therefore reduce the attraction of the path. The routes that are used frequently will have higher concentration of the pheromone trial and remain attractive. Thus, the shorter the route between the nest and food source imply short cycle time for the ants and these routes will have higher concentration of pheromone than the longer routes. Consequently, more ants are attracted by the shorter paths in the future. Finally, the shortest path will be discovered by the ant colony (Dorigo \& Stutzle, 2004; Zecchin et al., 2006).

In ACO algorithms, artificial ants are stochastic candidate solution construction procedures that exploit a pheromone model and possibly available heuristic information of the mathematical model. The artificial pheromone trails (numeric values) are the sole means of communication among the artificial ants. Pheromone decay, a mechanism analogous to the evaporation of the pheromone trial of the real ant colony allows the artificial ants to forget the past history and focus on new promising search directions. Like the natural ants, by updating the pheromone values according to the information learned in each of the preceding iterations, the algorithmic procedure leads to very good and hopefully, a global optimal solution. It was originally introduced to solve combinatorial optimization problems, in which decision variables are characterized by a finite set of components. However, in recent years, its adaptation to solve 
continuous (Liao et al., 2011, 2014, Socha \& Blum, 2007, Socha \& Dorigo, 2008) and mixed variable (Schluter et al., 2009, 2012, Socha, 2004) programming problems has received an increasing attention.

\subsection{Efficient Ant Colony Optimization (EACO) Algorithm}

One of the simplest and most widely used methods of random sampling is the Monte Carlo method. Monte Carlo method is a numerical method that provides approximate solution to a variety of physical and mathematical problems by random sampling. In crude Monte Carlo approach, a value is drawn at random from the probability distribution for each input, and the corresponding output value is computed. The entire process is repeated $\mathrm{n}$ times producing $\mathrm{n}$ corresponding output values. These output values constitute a random sample from the probability distribution over the output induced by the probability distributions over the inputs. The advantage of this approach is that the precision of the output distribution can be estimated using standard statistical techniques. The pseudorandom number generator produces samples that may be clustered in certain regions of the population and does not produce uniform samples. Therefore, in order to reach high accuracy, larger sample sizes are needed, which adversely affects the computational efficiency (Diwekar \& Kalagnanam, 1997; Diwekar \& Ulas, 2007). Gebreslassie \& Diwekar, (2015) proposed EACO algorithm that improves the performance of the conventional ACO algorithm for combinatorial, continuous and mixed variable optimization problems by introducing the Hammersley sequence sampling technique (HSS). The initial solution archive diversity for continuous and mixed-variable optimization problems plays an important role in the performance of ACO algorithm. The uniformity property of the HSS technique is exploited to avoid clustering of the initial solution archive in a small region of the potential solution space. Moreover, ACO algorithm is a probabilistic method; hence several random probability functions are involved in the algorithm procedure. For instance, for combinatorial ACO algorithm, the transition probability that help to choose the next solution component and for continuous and mixed-variable optimization problems, the probability of choosing ant guide from the solution archive to construct and sample the Gaussian kernel. The distribution of the random numbers generated for the acceptance probability of a solution component affects the performance of the ACO algorithm. At this stage, the multidimensional uniformity property of HSS is introduced to improve the computational efficiency of the ACO algorithm. The detail presentation of the EACO algorithms can be viewed (Gebreslassie \& 
274 Diwekar, 2015). The major steps in EACO algorithm are shown in Table 1 and the algorithm 275 proposed in this work that combines CAMD and EACO algorithm is given in Figure. 1.

276

277

278

279

280

281

282

283

284

285

286

287

288

289

290

291

292

293

\section{Results and Discussions}

\subsection{Solvent selection results}

The building block group indexes used in the case study are summarized in Appendix A Table A.2. The total number of groups used in this work is 24 and maximum of 10 groups per molecule are allowed. Therefore, the search space is composed of $24^{10}\left(6.34 * 10^{13}\right)$ combinations. The interaction parameter between the building groups and the three UNIFAC parameters: surface area, volume, and interaction parameters, as well as the boiling point parameters, the group free attachments and the molecular weights are tabulated in Appendix A Table A.1 and Table A.2, respectively.

The algorithm terminate if it reaches maximum number of iterations (MaxIter), or if the tolerance $(\epsilon)$ that is the relative difference between solutions found in two consecutive iterations is lower than or equal to the parameter $\epsilon$ for a set of consecutive number of iterations ICON. The algorithm parameters used to solve the problems are selected after performing a number of experimentations using different combination of the parameters. The algorithm parameters used for the EACO algorithm are the archive sizes $K=1500$, the number of ants $n A n t s=30, q=1 E-3$ and the tolerance $\epsilon=1 \mathrm{E}-6$, evaporation parameter $\rho=0.75$. 
Table 1: EACO Algorithm for mixed integer nonlinear optimization problems

\section{Start program}

- Set $K, n A n t s, N C, N O, N T, N O P T, \rho, q, \xi$ and termination criteria

- Initialize solution archive $T(K, N C+N O)$ using HSS

- Initialize solution archive $T(K, N T)$ randomly from the possible options

- Combine and evaluate the objective function of the k solutions $T(K, N D I M)$

- Rank solutions based on the quality of the objective function $\left(\mathrm{T}=\operatorname{rank}\left(S_{1} \ldots S_{K}\right)\right)$

- For categorical optimization problems, introduce multidimensional random number generated using HSS rand (IterMax $\times n A n t s \times N T, N O P T)$

\section{While Termination criterion is not satisfied}

- Generate solutions equivalent to the number of ants

For all \# nAnts

- Incremental solution construction

For all \# NDIM

○ Probabilistically construct continuous decision variables

- Probabilistically construct ordinal decision variables

○ Probabilistically construct categorical decision variables

\section{End for \# NDIM}

- Store and evaluate the objective function of the newly generated solutions

End for \# nAnts

- Combine, rank and select the best k solutions, $T=\operatorname{Best}\left(\operatorname{rank}\left(S_{1} \ldots S_{K} \ldots S_{K+n A n t}\right), K\right)$

- Update solution

\section{End while}

\section{End program}

$\mathrm{T}$ is solution archive and $\mathrm{k}$ is size of T. nAnts is the number of ants. NC, NO, NT and NDIM are the number of continuous, ordinal, categorical and the total number of decision variables, respectively. NOPT is the number of options in categorical variables. 


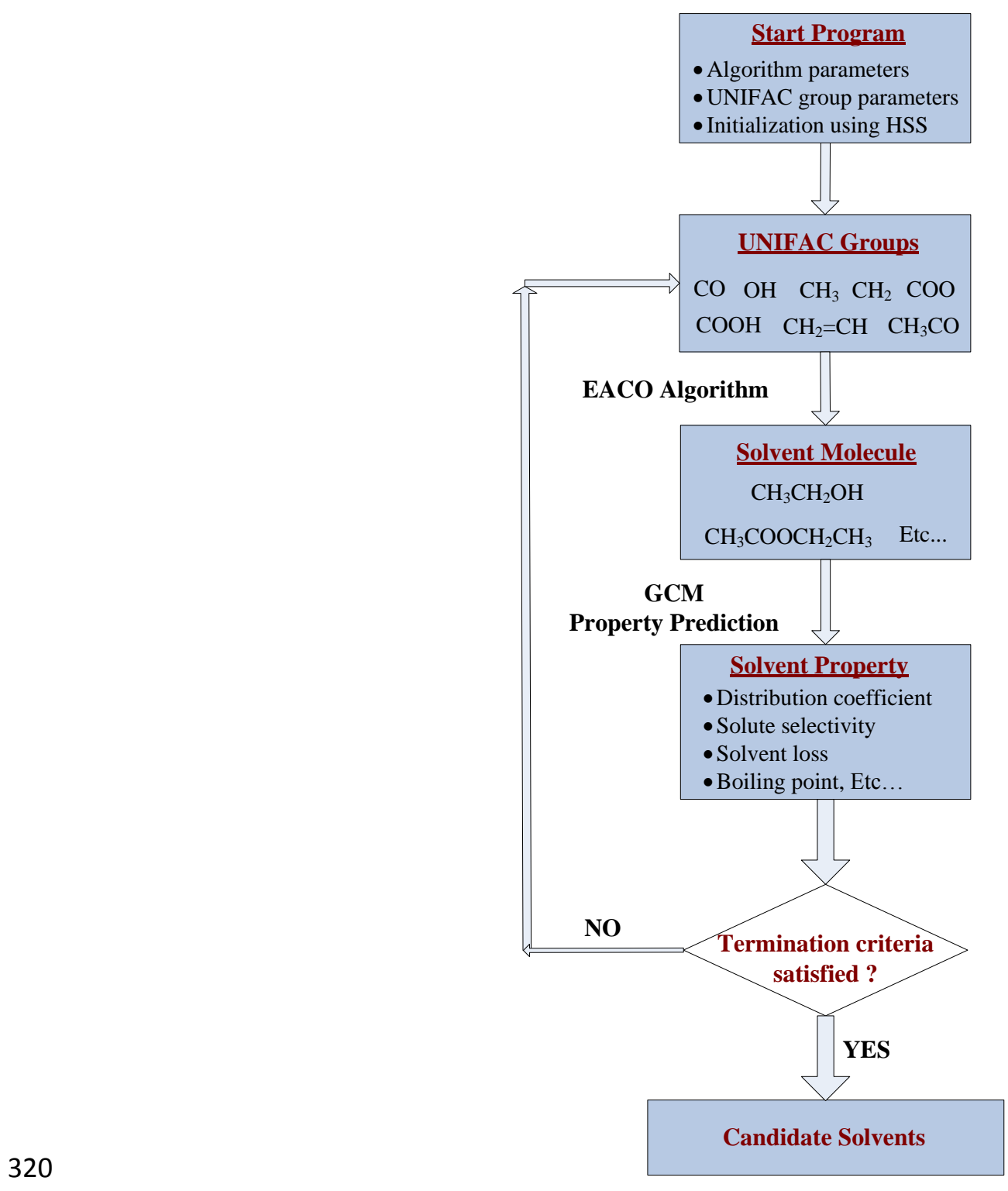

Figure 1: A basic algorithm for solvent selection using EACO.

322 An attractive solvent should have a high distribution coefficient, selectivity and low solvent 323 loss. In this case, the specifications are set to the above values in order to screen out the least 324 desirable candidates. The EACO algorithm generated more than 30 solvents which have higher 325 solute distribution value than the current practice of solvent (Ethyl acetate) for acetic acid 326 extraction, which also satisfy the given constraints as shown in Eqn. 11. The first top 15 solvents 327 for high boiling temperature solvents and the first top 7 low boiling temperature solvents are 328 summarized in Tables $2 \& 3$, respectively. As shown in the tables, most of the high ranked 329 solvent molecules are ethers, alcohols and aldehydes. Hostrup et al. (1999) has enlisted 7 
candidate solvent molecules for the extraction of acetic acid from water and among these molecules based on the environmental and toxicological properties of the candidates, 2 of the solvents (Diisobutylketone and Heptyl acetate) with low hazard were proposed as promising solvent substitutes for the removal of acetic acid from water. The solute distribution co-efficient of these 7 candidate solvents ranges from 0.11 (Methyl decanoate) to 0.5 (1-nonanol), which is relatively low compared to the result from this work that are enlisted in Tables $2 \& 3$. Among, the 7 candidate solvents only one (1-nonanol) would make the list. A high value of $m$ reduces the size of extraction equipment and the amount of recycling solvent. In other words, these high $\mathrm{m}$ solvents will reduce the investment cost and the operational cost related to the energy consumption of the solvent recovery. From Tables $2 \& 3$, it can be concluded that the EACO algorithm can generate a set of candidate solvents with a better solute distribution coefficient and low solvent loss properties. These candidate solvents may need further investigation related to environmental and toxicological properties and cost.

Comparing the results from EACO algorithm shown in Tables $2 \& 3$ and the results reported by Diwekar and co-workers (Kim \& Diwekar, 2002b, Xu \& Diwekar, 2005) for similar solvent selection problem obtained from heuristic algorithm; Efficient Simulated Annealing (ESA), the best solvent molecule found by EACO algorithm with configuration $\left(2 \mathrm{CH}_{2}, \mathrm{C}, \mathrm{CH}_{2}=\mathrm{CH}, \mathrm{CH}=\right.$ $\left.\mathrm{CH}, \mathrm{CH}_{3} \mathrm{OH}, \mathrm{CH}_{3} \mathrm{O}\right)$ has solute distribution $\mathrm{m}=0.691$, which exceeds the optimal solvent $\left(\mathrm{CH}_{3}\right.$, $6 \mathrm{CH}_{2}, \mathrm{OH}$ ) found by ESA with $\mathrm{m}=0.6074$. Moreover, the EACO algorithm has found a set of candidate solvent molecules with better solute distribution coefficients than the first ranked solvent molecule found by the ESA. There are two reasons for this phenomenon: (1) the EACO algorithm finds a set of solutions equal to the number of ants used by the algorithm instead of one as in the simulated annealing, and this property enables EACO algorithm the ability to cover the search space easily and gives a better chance to find the global optimum; and (2) In simulated annealing, an infeasible solution is accepted or discarded randomly according to the Metropolis criterion. The disadvantage of this constraint violation handling strategy is that there is no standard for accepting or discarding solutions. Therefore, some infeasible solutions that have more chances to reach the optimum may be discarded and, consequently, some good patterns are lost in the process. In EACO algorithm the selection of $q$ parameter is carefully chosen in such a way to focus initially at exploration and when the quality of the solution is improved to focus on exploitation and hence the probability of choosing the high ranked solutions in the solution 


\begin{tabular}{|c|c|c|c|c|c|}
\hline Rank & Solvent & $\mathrm{m}$ & $\beta$ & $\mathrm{S}_{\mathrm{L}}$ & $\mathrm{T}_{\mathrm{bp}}$ \\
\hline 1 & $2 \mathrm{CH}_{2}, \mathrm{C}, \mathrm{CH}_{2}=\mathrm{CH}, \mathrm{CH}=\mathrm{CH}, \mathrm{CH}_{3} \mathrm{OH}, \mathrm{CH}_{3} \mathrm{O}$ & 0.691 & 8.847 & 0.010 & 517.7 \\
\hline 2 & $\mathrm{CH}_{2}, \mathrm{CH}, \mathrm{C}, 2 \mathrm{CH}_{2}=\mathrm{CH}, \mathrm{CH}_{3} \mathrm{OH}, \mathrm{CH}_{3} \mathrm{O}$ & 0.683 & 8.660 & 0.009 & 509.7 \\
\hline 3 & $\mathrm{CH}_{3}, \mathrm{CH}, \mathrm{CH}_{2}-\mathrm{CH}, \mathrm{CH}_{2}=\mathrm{C}, \mathrm{C}=\mathrm{C}, \mathrm{CH}_{3} \mathrm{OH}, \mathrm{CH}_{3} \mathrm{O}$ & 0.628 & 8.060 & 0.006 & 539.6 \\
\hline 4 & $2 \mathrm{CH}_{3}, 3 \mathrm{CH}_{2}, \mathrm{CH}=\mathrm{C}, \mathrm{OH}$ & 0.615 & 11.388 & 0.008 & 455.9 \\
\hline 5 & $2 \mathrm{CH}_{3}, \mathrm{CH}_{2}, \mathrm{CH}=\mathrm{CH}, \mathrm{CH}=\mathrm{C}, \mathrm{OH}$ & 0.613 & 7.755 & 0.009 & 460.1 \\
\hline 6 & $2 \mathrm{CH}_{3}, \mathrm{CH}, 2 \mathrm{CH}=\mathrm{CH}, \mathrm{OH}$ & 0.609 & 7.585 & 0.008 & 459.7 \\
\hline 7 & $\mathrm{CH}_{3}, \mathrm{CH}_{2}=\mathrm{CH}, 2 \mathrm{CH}=\mathrm{C}, \mathrm{CH}_{3} \mathrm{OH}, \mathrm{CH}_{2} \mathrm{O}$ & 0.597 & 8.214 & 0.008 & 524.8 \\
\hline 8 & $2 \mathrm{CH}_{2}=\mathrm{CH}, \mathrm{CH}=\mathrm{C}, \mathrm{CH}_{3} \mathrm{O}, \mathrm{CH}_{2} \mathrm{O}$ & 0.580 & 8.080 & 0.005 & 424.8 \\
\hline 9 & $2 \mathrm{CH}_{2}=\mathrm{CH}, \mathrm{CH}_{2}=\mathrm{C}, \mathrm{CH}_{3} \mathrm{OH}, 2 \mathrm{CHO}$ & 0.566 & 7.966 & 0.005 & 531.5 \\
\hline 10 & $2 \mathrm{CH}_{2}, \mathrm{CH}=\mathrm{CH}, \mathrm{CH}=\mathrm{C}, \mathrm{CH}_{3} \mathrm{OH}$ & 0.517 & 9.129 & 0.009 & 452.6 \\
\hline 11 & $\mathrm{CH}_{3}, \mathrm{CH}_{2}=\mathrm{CH}, \mathrm{CH}=\mathrm{CH}, \mathrm{CH}_{3} \mathrm{CO}, \mathrm{CHO}$ & 0.451 & 7.241 & 0.006 & 459.3 \\
\hline 12 & $\mathrm{CH}_{3}, \mathrm{CH}, 2 \mathrm{CH}_{2}=\mathrm{C}, 2 \mathrm{CH}_{3} \mathrm{CO}$ & 0.448 & 12.003 & 0.009 & 528.7 \\
\hline 13 & $2 \mathrm{CH}_{2}, \mathrm{CH}=\mathrm{CH}, \mathrm{CH}_{2}=\mathrm{C}, 2 \mathrm{CH}_{3} \mathrm{CO}$ & 0.445 & 12.568 & 0.008 & 536.8 \\
\hline 14 & $\mathrm{CH}_{3}, 2 \mathrm{CH}=\mathrm{CH}, \mathrm{CH}_{3} \mathrm{CO}$ & 0.444 & 14.467 & 0.007 & 421.9 \\
\hline 15 & $3 \mathrm{CH}_{2}=\mathrm{C}, 2 \mathrm{CH}_{3} \mathrm{CO}$ & 0.437 & 9.397 & 0.008 & 525.7 \\
\hline
\end{tabular}

365

366

367

368

369

370

371

372

373

374

375

376

377

378

archive become higher because of the accumulation of pheromone dominates the evaporation as the quality of the solution increases. These two properties of EACO algorithm improves the efficiency of the search process and makes it less susceptible to being trapped in local optima.

Table 2: High boiling point temperature candidate solvents

Table 3: Low boiling point temperature candidate solvents

\begin{tabular}{clrrrr}
\hline Rank & \multicolumn{1}{c}{ Solvent } & $\mathrm{m}$ & \multicolumn{1}{c}{$\beta$} & \multicolumn{1}{c}{$\mathrm{S}_{\mathrm{L}}$} & $\mathrm{T}_{\mathrm{bp}}$ \\
\hline 1 & $\mathrm{CH}_{2}=\mathrm{CH}, \mathrm{CH}_{2}=\mathrm{C}, \mathrm{CH}_{3} \mathrm{O}, \mathrm{CH}_{2} \mathrm{O}$ & 0.751 & 7.79 & 0.033 & 374.9 \\
2 & $\mathrm{CH}_{2}=\mathrm{CH}, \mathrm{CH}=\mathrm{CH}, \mathrm{CH}_{3} \mathrm{O}$ & 0.709 & 9.25 & 0.019 & 337.2 \\
3 & $\mathrm{CH}_{3}, \mathrm{CH}_{2}=\mathrm{CH}, 2 \mathrm{CH}_{2} \mathrm{O}$ & 0.666 & 7.58 & 0.044 & 355.4 \\
4 & $\mathrm{CH}_{3}, \mathrm{CH}_{2}=\mathrm{C}, \mathrm{CH}_{3} \mathrm{CO}$ & 0.612 & 14.59 & 0.052 & 364.4 \\
5 & $\mathrm{CH}_{3}, \mathrm{CH}=\mathrm{CH}, \mathrm{CH}_{3} \mathrm{CO}$ & 0.610 & 16.10 & 0.048 & 372.0 \\
6 & $2 \mathrm{CH}_{3}, 2 \mathrm{CH}_{2}, \mathrm{COO}$ & 0.344 & 17.88 & 0.033 & 372.1 \\
7 & $2 \mathrm{CH}_{2}=\mathrm{CH}, \mathrm{COO}$ & 0.294 & 7.57 & 0.021 & 365.5 \\
\hline
\end{tabular}

Before discussing the results of the low boiling temperature solvents, the authors would like to acknowledge data discrepancy related to $\mathrm{HCOO}$ - building block. Excluding solvents that include HCOO-functional group, comparing Table 2 that enlists the low-boiling point candidate solvents found by EACO algorithm, and the low-boiling point candidate solvents found by ESA, the EACO algorithm found a better optimal solvent configuration $\left(\mathrm{CH}_{2}=\mathrm{CH}_{2} \mathrm{CH}_{2}\right.$ $\left.=\mathrm{C}, \mathrm{CH}_{3} \mathrm{O}, \mathrm{CH}_{2} \mathrm{O}\right)$ with $\mathrm{m}=0.751$ than the ESA with solvent configuration of $\left(\mathrm{CH}_{3}, \mathrm{CH}_{2}=\right.$ $\left.\mathrm{CH}, \mathrm{CH}_{3} \mathrm{O}, \mathrm{CH}-\mathrm{O}\right)$ and $\mathrm{m}=0.66$ and the EGA with solvent configuration of $\left(2 \mathrm{CH}_{3}, \mathrm{CH}_{2}, \mathrm{CH}_{2} \mathrm{CO}\right)$ and $m=0.5083$. We have observed that like EGA, the EACO algorithm does not find as many 
potential solvents as ESA. As explained by Xu and Diwekar (2005), this phenomena can be explained by the fact that in EACO algorithm, the q parameter focuses initially at exploration and as the quality of the solution improves, the algorithm focuses of the exploitation and hence the algorithm does not traverse as many local optima as ESA, which gives EACO a quicker convergence and a lesser probability of getting trapped in local optima. Moreover, in EACO algorithm, each ant constructs its own solution independently. Therefore, at each iteration, the different ant solution configurations leads to better solutions than only one solution configuration at each iteration like the ESA. This characteristics places EACO algorithm in a better position to cover the search space and find the global optimal solution than the ESA that may be trapped in local optima.

Moreover, the results from the EACO algorithm are also compared with the results of decomposition method proposed by Karunanithia et. al. (2005). In the decomposition method, the original MINLP problem is first reformulated into MILP master problem and NLP sub problems and solved using the gradient method solvers. It couples the integer solution from an MILP master problem and solution of an inner NLP sub-problem to solve the original MINLP formulation of the of CAMD problem (Eqn.11). Model parameters are first modified to adapt the solvent selection problem by Karunanithia et. al. (2005). The total number of UNIFAC groups used for the comparison is 16. Similar to the original model, maximum of 10 building blocks per molecule are allowed. Therefore, in this case the search space is composed of $16^{10}(1.099 \mathrm{E}+12)$ combinations. The parameters used for the EACO algorithm are the archive sizes $\mathrm{K}=1500$, number of ants $\mathrm{nAnt}=30$, algorithm parameter $\mathrm{q}=1 \mathrm{E}-3$ and tolerance $\epsilon=1 \mathrm{E}-6$. The property constraints such as the lower bound of the boiling point, solute distribution, and selectivity are $340 \mathrm{~K}, 0.49$ and 11 , respectively and the upper bound of the solvent loss is 0.0038 as given in Karunanithia et. al. (2005). The results are tabulated in Table 4. As shown in the table, in terms of the desired thermodynamic properties of solvents, the solvents generated using the EACO algorithm are superior to the 2-hexananone solvent proposed from the decomposition method by Karunanithia et. al. (2005). The proposed solution strategy provides solvents with better thermodynamic properties and from this it can be concluded that the proposed methodology can be a useful alternative to optimization of large scale CAMD problems. 
Table 4: EACO algorithm compared to decomposition method Karunanithia et. al. (2005).

\begin{tabular}{lccccc}
\hline \multicolumn{1}{c}{ Method } & Solvent & $\mathrm{m}$ & $\beta$ & $\mathrm{S}_{\mathrm{L}}$ & $\mathrm{T}_{\mathrm{bp}}$ \\
\hline Karunanithi et. al. (2005) & $1 \mathrm{CH} 3,3 \mathrm{CH} 2,1 \mathrm{CH} 3 \mathrm{CO}$ & 0.491 & 11 & 0.0038 & 404.1 \\
EACO & $3 \mathrm{CH} 3, \mathrm{CH} 2, \mathrm{C}, \mathrm{CH} 3 \mathrm{COO}$ & 0.533 & 11.8 & 0.0036 & 463.2 \\
EACO & $2 \mathrm{CH} 3,3 \mathrm{CH} 2, \mathrm{CH} 2 \mathrm{O}, \mathrm{CH} 2 \mathrm{COO}$ & 0.507 & 39.2 & 0.0037 & 414.7
\end{tabular}

The CAMD optimization problem results from EACO and the conventional ACO algorithms to the high and low boiling point temperature solvents are presented in Tables $5 \& 6$. The 412 parameters used for the $\mathrm{ACO}$ algorithms are 2 archive sizes $(\mathrm{K}=500$, and $\mathrm{K}=1000)$, $\mathrm{nAnts}=$ $41330, q=1 \mathrm{E}-3, \epsilon=1 \mathrm{E}-6$ and $\rho=0.75$. As shown in the tables, on all cases, the performance of the 414 EACO algorithm out performs the conventional ACO algorithm and the performance 415 improvement ranges from $23.7 \%$ to $52.9 \%$. The performance improvement is because of the 416 multidimentional uniformity property of HSS, the EACO algorithm needs less iteration than the 417 conventional ACO to find the optimal candidate solvents.

Table 5: High boiling point temperature solvent.

\begin{tabular}{cccccc}
\hline \multirow{2}{*}{$\boldsymbol{K}$} & \multicolumn{2}{c}{ EACO } & \multicolumn{2}{c}{ ACO } & Improve \\
\cline { 2 - 5 } & $\mathrm{m}$ & Iter & $\mathrm{m}$ & Iter & {$[\%]$} \\
\hline 500 & 0.61 & 108 & 0.62 & 153 & 29.4 \\
1000 & 0.61 & 64 & 0.62 & 136 & 52.9 \\
\hline
\end{tabular}

Table 6: Low boiling point temperature solvent.

\begin{tabular}{cccccc}
\hline \multirow{2}{*}{$\boldsymbol{K}$} & \multicolumn{2}{c}{ EACO } & \multicolumn{2}{c}{ ACO } & Improve \\
\cline { 2 - 5 } & $\mathrm{m}$ & Iter & $\mathrm{m}$ & Iter & {$[\%]$} \\
\hline 500 & 0.75 & 29 & 0.75 & 38 & 23.7 \\
1000 & 0.71 & 62 & 0.71 & 87 & 28.7 \\
\hline
\end{tabular}

421 Sample of the convergence path of the EACO versus the conventional ACO for the CAMD 422 problem is given in Figure 2. The figure presents the trajectories of the solute distribution 423 coefficient of the CAMD optimization problem as a function of the number of iterations to reach 424 the optimal solution. As shown in the figure, the EACO algorithm found the optimal solution at $42568^{\text {th }}$ iteration. However, the conventional ACO needs 85 iterations to reach a local optimal value. 426 Moreover, as shown in the figure, the EACO finds a feasible solution at $5^{\text {th }}$ iteration while the 
427

conventional ACO finds the first feasible solution at the $11^{\text {th }}$ iteration. All the above observations prove that EACO algorithm benefits from the uniformity property of HSS.

\subsection{Ordinal vs Categorical EACO algorithms to solve CAMD}

Depending on the type of the discrete decision variables (i.e. ordinal and categorical variables), there are two approaches for solving the combinatorial optimization problems in the EACO algorithm (Gebreslassie and Diwekar 2015). In the case of ordinal variables, the problem can be tackled through relaxation of the discrete variables, where in CAMD problem represents the index of the UNIFAC building block groups. Therefore, it can be solved by including a heuristic rule to the original algorithm for continuous variable optimization problem. The CAMD problem also can be solved using the EACO algorithm for optimization problems that involve categorical variables. To demonstrate the performance difference between these two approaches, results for high boiling point and low boiling point temperature solvents are presented in Tables $7 \& 8$. The parameters used for the EACO algorithm are archive sizes $K=500, n A n t s=30, q=$ $1 E-3$ and tolerance $\epsilon=1 E-6$, evaporation parameter $\rho=0.75$. As shown in the tables, the performance of the EACO algorithm for categorical variables outperforms the EACO algorithm for ordinal variable interims of the number of iterations needed to reach the optimal solution. Iteration improves by $65.7 \%$ for low boiling temperature and $70 \%$ for high boiling temperature solvents. Moreover, the quality of the solution using the EACO algorithm for categorical variables is better for high boiling temperature solvents. As far as for the low boiling temperature solvents EACO algorithm for ordinal discrete variables performs better.

Socha (2009) reported that ACO algorithm for ordinal variables perform better on problems containing discrete variables that can be ordered and ACO algorithm for optimization problems that involve categorical variables perform better on problems where a proper ordering is not possible, or unknown (categorical variables). The CAMD optimization problem only involves discrete variables that can be ordered. However, the results show that in most case the performance of the EACO algorithm for categorical variables perform better than the algorithm for ordinal variables. 


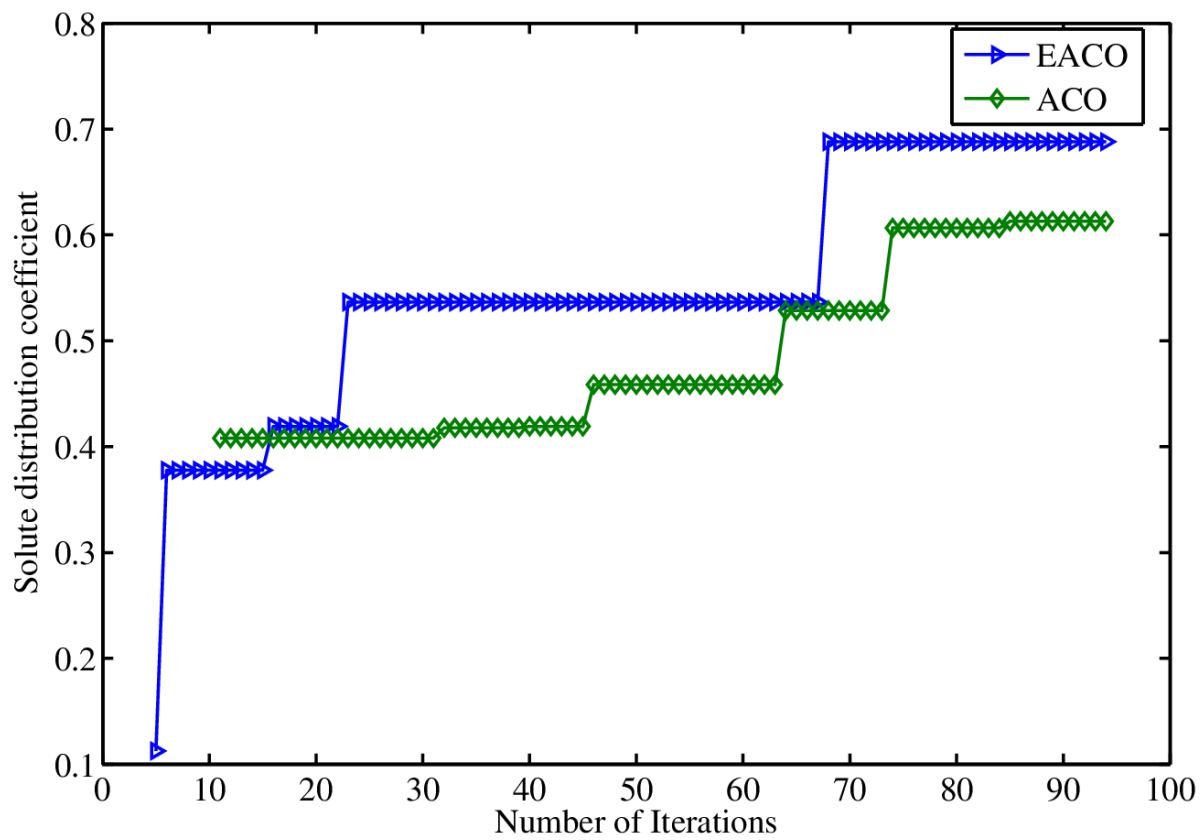

Figure 2: EACO vs ACO algorithm trajectory of solving CAMD optimization problem.

Table 7: High boiling point temperature: Algorithm for ordinal vs categorical

\begin{tabular}{lcccc}
\hline & Iter & Improve [\%] & $\mathrm{m}$ & Improve [\%] \\
\hline Ordinal & 108 & 0.0 & 0.613 & 0.0 \\
Categorical & 37 & 65.7 & 0.615 & 0.3 \\
\hline
\end{tabular}

Table 8: Low boiling point temperature: Algorithm for ordinal vs categorical

\begin{tabular}{lcccc}
\hline & Iter & Improve [\%] & $\mathrm{m}$ & Improve [\%] \\
\hline Ordinal & 20 & 0.0 & 0.751 & 5.9 \\
Categorical & 6 & 70.0 & 0.709 & 0.0 \\
\hline
\end{tabular}

\section{Conclusions}

This paper proposes a new alternative optimization strategy based on metaheuristic EACO algorithm to solve computer-aided molecular design problems. The proposed methodology involves formulating the solvent selection molecular design problem as an MINLP model and solution method for identifying candidate solvents and proposing the optimal solvent molecule.

The ACO algorithm is a simple to implement and yet an effective optimization framework for handling combinatorial, continuous and mixed-variable optimization problems. In this work, the EACO algorithm as an alternative to the gradient based, simulated annealing and genetic algorithm optimization is implemented to solve CAMD problems. A real world case study of 
solvent selection for acetic acid extraction from process waste stream is presented and discussed in this work. The GCM technique is implemented to estimate the mixture properties and using EACO algorithm, new solvents with better targeted properties are proposed. The EACO algorithm has found a set of candidate solvent molecules with better solute distribution than the first ranked solvent molecules proposed using ESA and the decomposition methods.

\section{Acknowledgements}

We gratefully acknowledge the funding from DOE National Energy Laboratory grant \# DEFE0012451.

\section{References}

Achenie, L. E. K., \& Sinha, M. (2003). Interval global optimization in solvent design. Reliable Computing, 9, 317-38.

Alvarado-Morales, M., Terra, J., Gernaey, K.V., Woodley, J.M., and R. Gani R. (2009). Biorefining: Computer aided tools for sustainable design and analysis of bioethanol production. Chemical Engineering Research and Design, 87, 1171-1183

Chemmangattuvalappil, N.G, Eljack, F.T, Solvason, C.C, Eden, M.R., (2009) A novel algorithm for molecular synthesis using enhanced property operators. Computers and Chemical Engineering, 33, 636-643.

Cheng, H.C., \& Wang, F.S. (2008). Optimal biocompatible solvent design for a two-stage extractive fermentation process with cell recycling. Computers \& Chemical Engineering, 32, 1385-96.

Diwekar, U., \& Xu, W. (2005). Improved genetic algorithms for deterministic optimization and optimization under uncertainty. Part I. algorithms development. Industrial and Engineering Chemistry Research, 44, 7132-7.

Diwekar, U. M., \& Kalagnanam, J. R. (1997). Efficient sampling technique for optimization under uncertainty. AIChE Journal, 43, 440-7.

Diwekar, U. M., \& Shastri, Y. (2011). Design for environment: a state-of-the-art review. Clean Technology \& Environmental Policy, 13, 227-40.

Diwekar, U. M., \& Ulas, S. (2007). Sampling Techniques volume 26. Encyclopedia of Chemical Technology.

Dorigo, M. (1992). Optimization, learning and natural algorithms. PhD Thesis, Dept. of Electronics, Politecnico di Milano, Italy.

Dorigo, M., \& Stutzle, T. (2004). Ant colony optimization theory. A Brandford Book, The MIT Press, Cambridge, Massachusetts.

Eljack, F. T., \& Eden, M. R. (2008). Systematic visual approach to molecular design via property clusters and group contribution methods. Computers \& Chemical Engineering, 32, 300210 . 
Gebreslassie, B. H., \& Diwekar, U. M. (2015). Efficient ant colony optimization (EACO) algorithm for deterministic optimization, to be submitted.

Gernaey K. V., and Gani, R. (2010). A model-based systems approach to pharmaceutical product process design and analysis. Chemical Engineering Science, 65, 5757-5769.

Giovanoglou A., Barlatier J., Adjiman C.S, Pistikopoulos E.N., \& Cordiner, J. L. (2003). Optimal solvent design for batch separation based on economic performance. AIChE Journal, 49, 3095-109.

Hansen, H. K., Rasmussen, P., Fredenslund, A., Schiller, M., \& Gmehling J. (1991). Vaporliquid equilibria by UNIFAC group contribution. Industrial \& Engineering Chemistry Research, 30, 2352-5.

Harper, P., Gani, R., Kolar, P., \& Ishikawa, T. (1999). Computer-aided molecular design with combined molecular modeling and group contribution. Fluid Phase Equilibria, 158-160, 337-47.

Harper, P. M., \& Gani, R. (2000). A multi-step and multi-level approach for computer aided molecular design. Computers \& Chemical Engineering, 24, 677-83.

Holland, J. H. (1975). Adaptation in natural and artificial systems: An introductory analysis with applications to biology, control, and artificial intelligence. University of Michigan press: Ann Arbor, MI.

Harper, P., \& Gani, R. (1999). Design of environmentally benign processes: Integration of solvent design and separation process synthesis. Computers \& Chemical Engineering, 23, $1395-414$.

Joback, K.G. \& Reid R.C. (1987). Estimation of pure-component properties from groupcontributions. Chemical Engineering Communications, 57, 233-43.

Karunanithi, A. T., Achenie, L. E. K., \& Gani, R. (2006). A computer-aided molecular design framework for crystallization solvent design. Chemical Engineering Science, 61, 1247-60.

Karunanithi, A. T., Achenie, L. E. K., \& Gani, R. (2005). A new decomposition-based computeraided molecular/mixture design methodology for the design of optimal solvents and solvent mixtures. Industrial \& Engineering Chemistry Research, 4, 4785-97.

Kazantzi, V., Qin, X., El-Halwagi, M., Eljack F.T and Eden M.R., (2007) Simultaneous Process and Molecular Design through Property Clustering. Ind. Eng. Chem. Res, 46, 3400-3409.

Kim, K., \& Diwekar, U. (2002a). Efficient combinatorial optimization under uncertainty. 1. Algorithmic development. Industrial and Engineering Chemistry Research, 41, 1276-84.

Kim, K., \& Diwekar, U. (2002b). Efficient combinatorial optimization under uncertainty. 2. Application to stochastic solvent selection. Industrial and Engineering Chemistry Research, 41, 1285-96.

Kim, K., \& Diwekar, U. (2002c). Hammersley stochastic annealing: Efficiency improvement for combinatorial optimization under uncertainty. IIE Transactions Institute of Industrial Engineers, 34, 761-77.

Kirkpatrick, S., Gelatt, C. D., \& Vecchi, M. P. (1983). Optimization by simulated annealing. Science, 220, 671-80.

Li, M., Harten, P. F., \& Cabezas, H. (2002). Experiences in designing solvents for the environment. Industrial \& Engineering Chemistry Research, 41, 5867-77. 
Liao, T., Montes De Oca, M.A., Aydin, D., Stutzle, T., \& Dorigo, M. (2011). An incremental ant colony algorithm with local search for continuous optimization. (pp. 125-32).

Liao, T., Stutzle, T., Montes De Oca, M.A., \& Dorigo, M. (2014). A unified ant colony optimization algorithm for continuous optimization. European Journal of Operational Research, 234, 597-609.

Marrero J. \& Gani R. (2001). Group - contribution based estimation of pure component properties. Fluid Phase Equilibria, 184, 183-208.

Odele, O. and Macchietto S. (1993). Computer aided molecular design: a novel method for optimal solvent selection. Fluid Phase Equilibria, 82, 47-54.

Ostrovsky, G. M., Achenie L.E.K., \& Sinha, M. (2002). On the solution of mixed-integer nonlinear programming models for computer aided molecular design. Computers and Chemistry, 26, 645-60.

Samudra A. P and Sahinidis N.V., (2013) Optimization-Based Framework for Computer-Aided Molecular Design. AIChE Journal, 59(10), 3686-3701.

Satyanarayana, K.C., Abildskov, J. and Gani R., (2009) Computer-aided polymer design using group contribution plus property models. Computers and Chemical Engineering, 33, 10041013.

Schluter, M., \& Gerdts, M. (2010). The Oracle penalty method. Journal of Global Optimization, 47, 293-325.

Schluter, M., Gerdts, M., \& Ruckmann, J.J. (2012). A numerical study of MIDACO on 100 MINLP benchmarks. Optimization, 61, 873-900.

Socha, K. (2004). ACO for continuous and mixed-variable optimization. Lecture Notes in Computer Science (including subseries Lecture Notes in Artificial Intelligence and Lecture Notes in Bioinformatics), 3172 LNCS, 25-36.

Socha, K. (2009). Ant Colony Optimization for Continuous and Mixed-Variable Domains. PhD Thesis, IRIDIA, CoDE, Universit'e Libre de Bruxelles, CP 194/6.

Socha, K., \& Blum, C. (2007). An ant colony optimization algorithm for continuous optimization: Application to feed-forward neural network training. Neural Computing and Applications, 16, 235-47.

Socha, K., \& Dorigo, M. (2008). Ant colony optimization for continuous domains. European Journal of Operational Research, 185, 1155-73.

$\mathrm{Xu}, \mathrm{W} .$, \& Diwekar, U. (2005). Improved genetic algorithms for deterministic optimization and optimization under uncertainty. Part II. Solvent selection under uncertainty. Industrial and Engineering Chemistry Research, 44, 7138-46.

Yamamoto, H., \& Tochigi, K. (2008). Computer-aided molecular design to select foaming agents using a neural network method. Industrial \& Engineering Chemistry Research, 47, 5152-6.

Zecchin, A., Simpson, A., Maier, H., Leonard, M., Roberts, A., \& Berrisford, M. (2006). Application of two ant colony optimization algorithms to water distribution system optimization. Mathematical and Computer Modelling, 44, 451-68. 


\section{Appendix A. Parameters}

Table A.1: Interaction parameters between main group m and n $\left(a_{m n}\right)$

\begin{tabular}{crrrrrrrrrrrr}
\hline $\mathrm{m} \backslash \mathrm{n}$ & \multicolumn{1}{c}{1} & \multicolumn{1}{c}{2} & \multicolumn{1}{c}{3} & \multicolumn{1}{c}{5} & \multicolumn{1}{c}{5} & \multicolumn{1}{c}{6} & 7 & \multicolumn{1}{c}{8} & \multicolumn{1}{c}{9} & 10 & 11 \\
\hline 1 & 0 & 86.02 & 986.5 & 697.2 & 1318 & 476.4 & 677 & 232.1 & 507 & 251.5 & 663.5 & 387.1 \\
2 & -35.36 & 0 & 524.1 & 787.6 & 270.6 & 182.6 & 448.8 & 37.85 & 333.5 & 214.5 & 318.9 & 48.33 \\
3 & 156.4 & 457 & 0 & -137.1 & 353.5 & 84 & -203.6 & 101.1 & 267.8 & 28.06 & 199 & 190.3 \\
4 & 16.51 & -12.52 & 249.1 & 0 & -181 & 23.39 & 306.4 & -10.72 & 179.7 & -128.6 & -202 & 165.7 \\
5 & 300 & 496.1 & -229.1 & 289.6 & 0 & -195.4 & -116 & 72.87 & 0 & 540.5 & -14.09 & -197.5 \\
6 & 26.76 & 42.92 & 164.5 & 108.7 & 472.5 & 0 & -37.36 & -213.7 & -190.4 & -103.6 & 669.4 & -18.8 \\
7 & 505.7 & 56.3 & 529 & -340.2 & 480.8 & 128 & 0 & -110.3 & 766 & 304.1 & 497.5 & 0 \\
8 & 114.8 & 132.1 & 245.4 & 249.6 & 200.8 & 372.2 & 185.1 & 0 & -241.8 & -235.7 & 660.2 & 560.2 \\
9 & 329.3 & 110.4 & 139.4 & 227.8 & 0 & 385.4 & -236.5 & 1167 & 0 & -234 & -268.1 & -122.3 \\
10 & 83.36 & 26.51 & 237.7 & 238.4 & -314.7 & 191.1 & -7.84 & 461.3 & 457.3 & 0 & 664.6 & 417 \\
11 & 315.3 & 1264 & -151 & 339.8 & -66.17 & -297.8 & -165.5 & -256.3 & 193.9 & -338.5 & 0 & -337 \\
12 & 529 & 1397 & 88.63 & 171 & 284.4 & 123.4 & 577.5 & -234.9 & 145.4 & -247.8 & 1179 & 0 \\
\hline
\end{tabular}


Table A.2: The surface area $R_{k}$ and volume $Q_{k}$ values for the UNIFAC equation, boiling point parameters $t_{a}$, free attachments $b_{i}$ and molecular weight Mw

\begin{tabular}{cccrrrrr}
\hline $\begin{array}{c}\text { MG } \\
\text { index }\end{array}$ & $\begin{array}{c}\text { Sup } \\
\text { groups }\end{array}$ & $\begin{array}{c}\text { SG } \\
\text { index }\end{array}$ & \multicolumn{1}{c}{$\mathrm{R}_{\mathrm{k}}$} & \multicolumn{1}{c}{$\mathrm{Q}_{\mathrm{k}}$} & \multicolumn{1}{c}{$\mathrm{t}_{\mathrm{a}}$} & $\mathrm{b}_{\mathrm{i}}$ & $\mathrm{MW}$ \\
\hline 1 & $\mathrm{CH}_{3}-$ & 1 & 0.9011 & 0.848 & 23.58 & 1 & 15 \\
1 & $-\mathrm{CH}_{2}-$ & 2 & 0.6744 & 0.54 & 22.88 & 2 & 14 \\
1 & $-\mathrm{CH}<$ & 3 & 0.4469 & 0.228 & 21.74 & 3 & 13 \\
1 & $>\mathrm{C}<$ & 4 & 0.2195 & 0 & 18.25 & 4 & 12 \\
2 & $\mathrm{CH}_{2}=\mathrm{CH}-$ & 5 & 1.3454 & 1.176 & 43.14 & 1 & 27 \\
2 & $-\mathrm{CH}=\mathrm{CH}-$ & 6 & 1.1167 & 0.867 & 49.92 & 2 & 26 \\
2 & $\mathrm{CH}_{2}=\mathrm{C}<$ & 7 & 1.1173 & 0.988 & 42.32 & 2 & 26 \\
2 & $-\mathrm{CH}=\mathrm{C}<$ & 8 & 0.8886 & 0.676 & 49.1 & 3 & 25 \\
2 & $>\mathrm{C}=\mathrm{C}<$ & 9 & 0.6605 & 0.485 & 48.28 & 4 & 24 \\
3 & $-\mathrm{OH}$ & 10 & 1 & 1.2 & 92.88 & 1 & 17 \\
4 & $\mathrm{CH}{ }_{3} \mathrm{OH}$ & 11 & 1.4311 & 1.432 & 116.46 & 0 & 32 \\
5 & $\mathrm{H}_{2} \mathrm{O}$ & 12 & 0.92 & 1.4 & 175.03 & 0 & 18 \\
6 & $\mathrm{CH}_{3} \mathrm{CO}-$ & 13 & 1.6724 & 1.488 & 100.33 & 1 & 43 \\
6 & $-\mathrm{CH}_{2} \mathrm{CO}-$ & 14 & 1.4457 & 1.18 & 99.63 & 1 & 42 \\
7 & $-\mathrm{CHO}$ & 15 & 0.998 & 0.948 & 74.74 & 1 & 29 \\
8 & $\mathrm{CH}_{3} \mathrm{COO}-$ & 16 & 1.9031 & 1.728 & 104.68 & 1 & 59 \\
8 & $-\mathrm{CH}_{2} \mathrm{COO}-$ & 17 & 1.6764 & 1.42 & 103.98 & 2 & 58 \\
9 & $\mathrm{HCOO}_{-}$ & 18 & 1.242 & 1.188 & 84.88 & 1 & 45 \\
10 & $\mathrm{CH}_{3} \mathrm{O}-$ & 19 & 1.145 & 1.088 & 46 & 1 & 31 \\
10 & $-\mathrm{CH}{ }_{2} \mathrm{O}-$ & 20 & 0.9183 & 0.78 & 45.3 & 2 & 30 \\
10 & $>\mathrm{CH}-\mathrm{O}-$ & 21 & 0.6908 & 0.468 & 44.16 & 3 & 29 \\
11 & $-\mathrm{COOH}$ & 22 & 1.3013 & 1.224 & 160.8 & 1 & 45 \\
11 & $\mathrm{HCOOH}_{12}$ & 23 & 1.528 & 1.532 & 175.53 & 0 & 46 \\
12 & $-\mathrm{COO}-$ & 24 & 1.38 & 1.2 & 81.1 & 2 & 44 \\
\hline & & & & & & &
\end{tabular}

